\title{
Secure eHealth: A Secure eHealth System to Detect COVID Using Image Steganography
}

جامعة قطر QATAR UNIVERSITY
Nandhini Subramanian, Omar Elharrouss, Somaya Al-Maadeed,

Department of Computer Science and Engineering,

Qatar University

\section{Introduction}

COVID is a pandemic which has spread to all parts of the world.

Detection of COVID infection is crucial to prevent the spread further. Contactless health care systems are essential which can be implemented with Cloud computing.

Privacy and security of the medical image data transferred through untrusted channels cannot be ensured.

The main aim is to secure the medical details when transferring them from the end device to the cloud and vice versa using image steganography.

The medical lung images are masked under a normal and natural cover images.

\section{Research Questions}

1. Can deep learning methods produce secure and robust image steganography without complicated structure?

2. Is it possible to hide a RGB secret image of the same size as the RGB cover image without any distortion?

\section{System Overview}

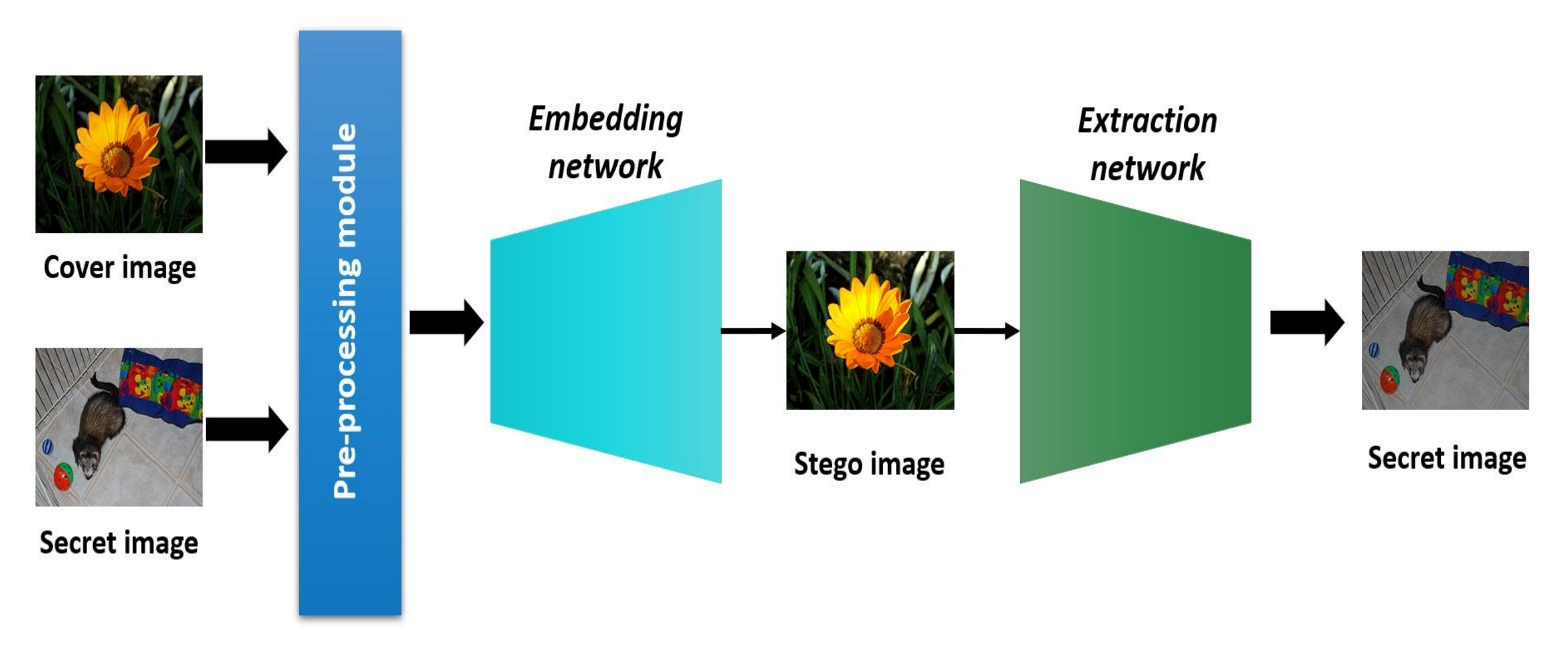

\section{Methodology}

Preprocessing Module : Three layers of convolutional layer. Both the original cover image and original secret image are passed concurrently to extract features. A concatenation layer is used to merge the features.

Embedding Network: The merged features are given as input to reconstruct the steganography image with secret image embedded in the cover image. Embedding Network is situated at the end devices side. Pre-processing and the embedding are carried out together

Extraction Network: The steganography image is used to input to extract the secret image. Extraction network is at the Cloud Server end.

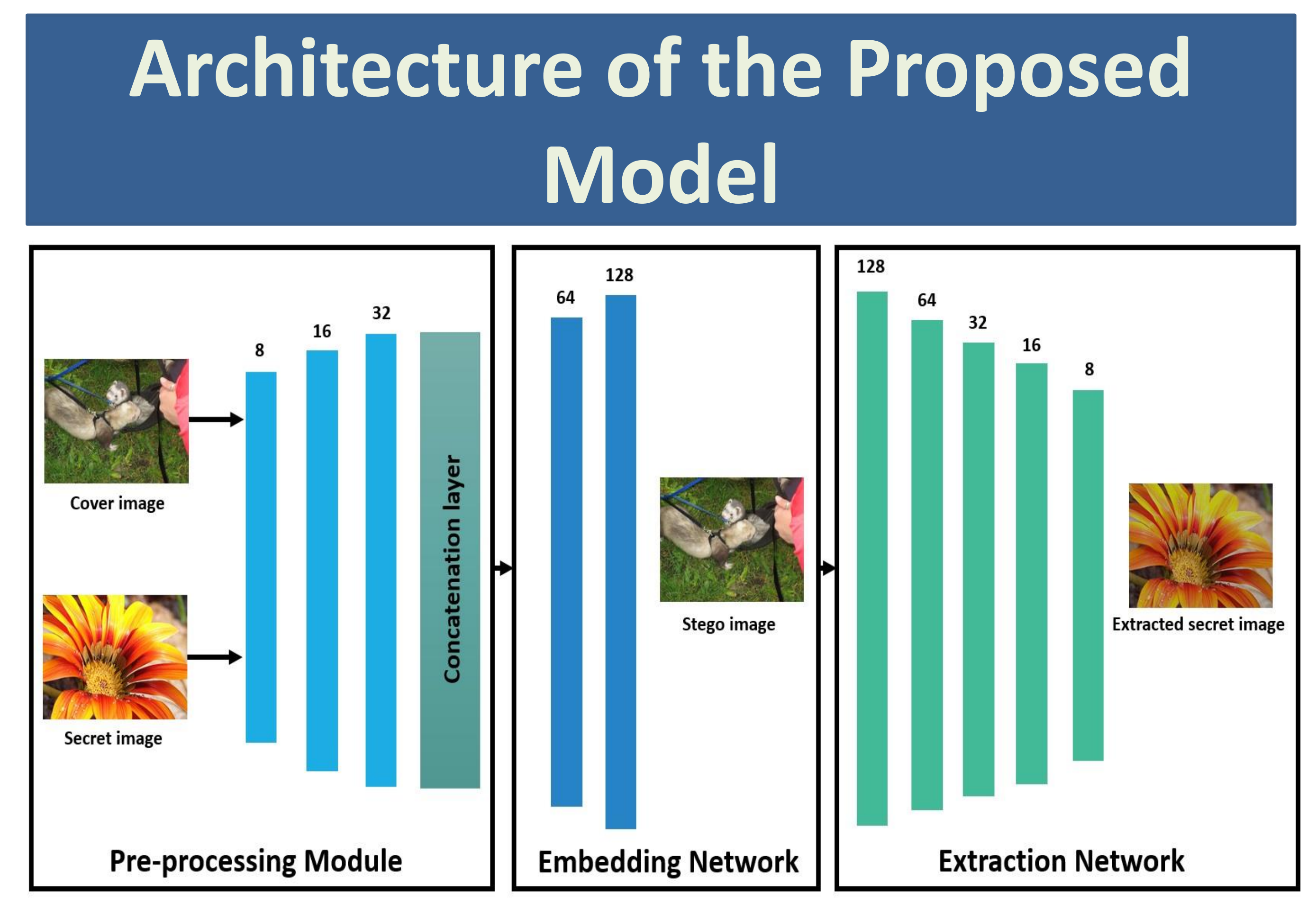

\section{Tiny Imagenet - Sample Images}
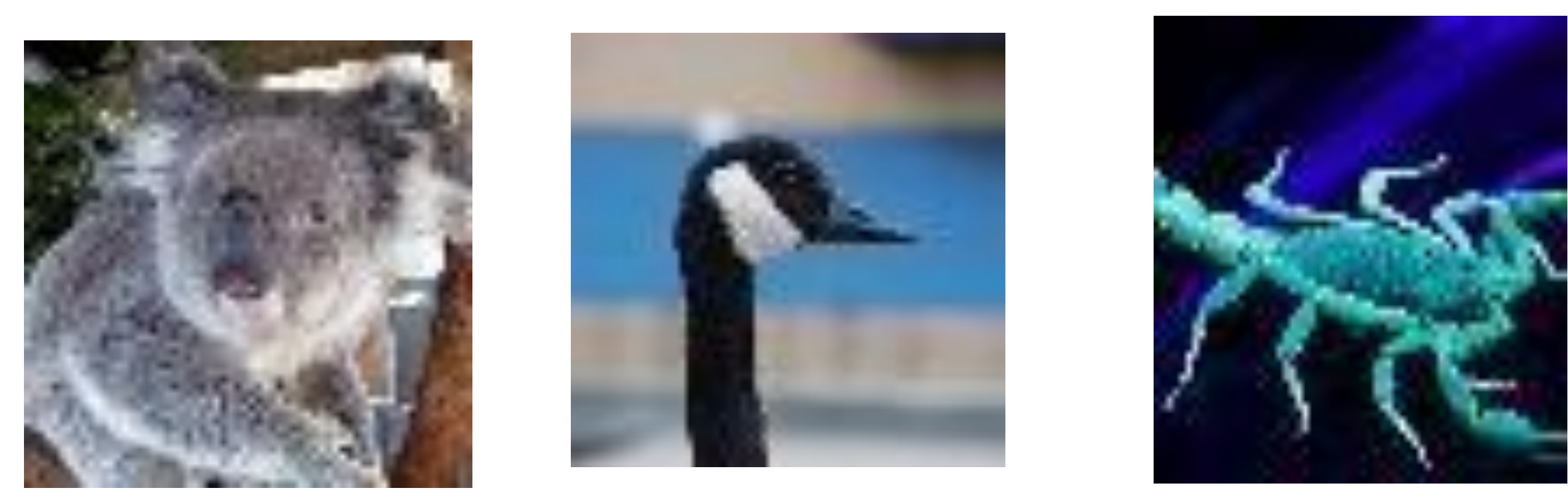

\section{Evaluation Metrics}

$$
\begin{gathered}
M S E=\frac{\sum_{R, C}\left[\operatorname{Image}_{1}(r, c)-\operatorname{Image}_{2}(r, c)\right]^{2}}{R * C} \\
P S N R=10 L O G_{10}\left(\frac{M^{2}}{M S E}\right)
\end{gathered}
$$

\section{Results}

\section{Result of the Proposed Method}

\begin{tabular}{|c|c|c|c|c|}
\hline Method & \multicolumn{2}{|c|}{ Embedding Network } & \multicolumn{2}{|c|}{ Extraction Network } \\
\cline { 2 - 5 } & MSE & PSNR & MSE & PSNR \\
\hline $\begin{array}{c}\text { Proposed } \\
\text { Method }\end{array}$ & 77.18 & 29.30 & 44.85 & 27.90 \\
\hline
\end{tabular}

\section{Comparison Results with Traditional} Steganography Methods

\begin{tabular}{|c|c|c|c|c|c|}
\hline Method & $\begin{array}{c}\text { Image } \\
\text { Size }\end{array}$ & Airplane & Baboon & Lena & Peppers \\
\hline$[\mathbf{1}]$ & $512 \times 512$ & 37 & 37 & 37 & 37 \\
\hline$[\mathbf{2}]$ & $512 \times 512$ & 32.74 & 32.41 & 33.16 & 32.41 \\
\hline$[3]$ & $512 \times 512$ & - & - & 32.09 & - \\
\hline$[4]$ & $512 \times 512$ & 32.774 & 32.448 & 32.49 & 32.725 \\
\hline $\begin{array}{c}\text { Proposed } \\
\text { Method }\end{array}$ & $64 \times 64$ & 32.15 & 29.76 & 29.49 & 29.14 \\
\hline
\end{tabular}

Image result of the Proposed Method on ImageNet dataset
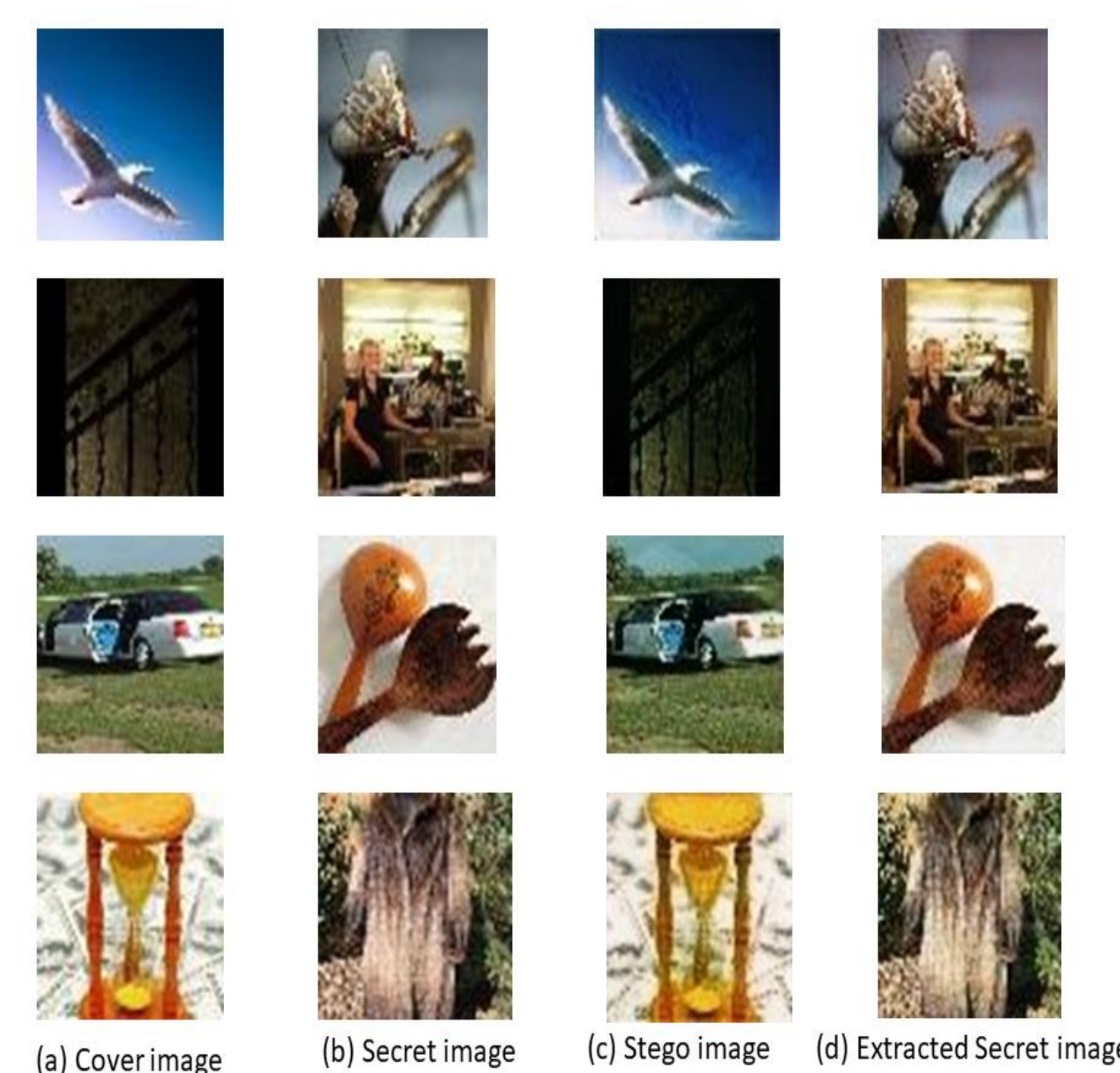

Image result of the Proposed Method on real time lung $X$-ray images

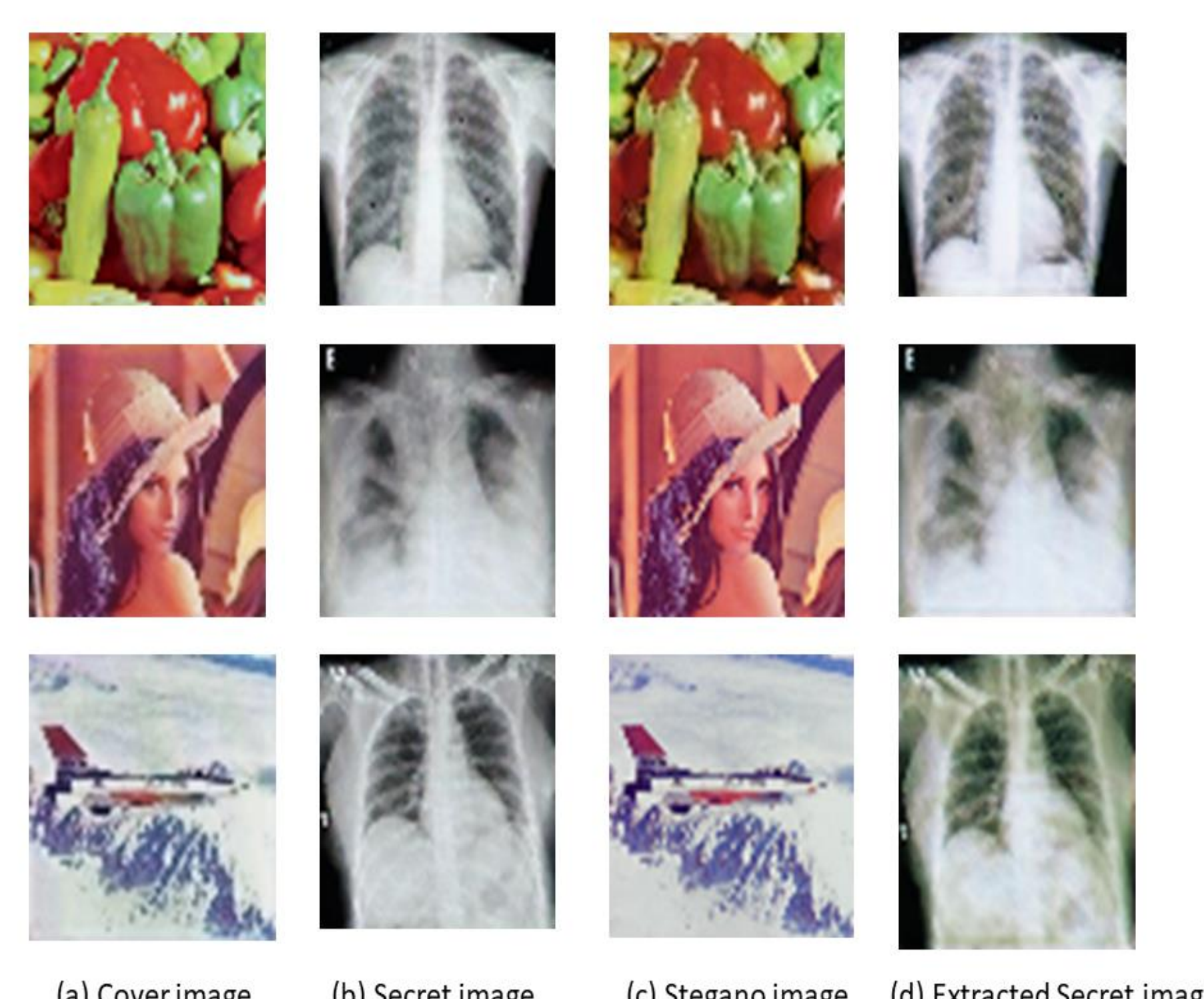

\section{Discussion}

It can be clearly seen that the generated steganography image obtained using the proposed deep learning model is similar to the cover image without any noise and higher invisibility.

Generated steganography images have higher invisibility, higher security and robustness compared to the traditional LSB methods.

Higher security and robustness is obtained even when the cover image is plain (black/white) and the secret image is full of information.

Privacy and security of the medical records of the patients is ensured through image steganography.

\section{Acknowledgment}

This work was made possible by NPRP11S-0113180276 from the Qatar National Research Fund (a member of Qatar Foundation). The findings achieved herein are solely the responsibility of the author.

\section{Contact}

Nandhini Subramanian

Email:ns1808900@student.qu.edu.qa

\section{References}

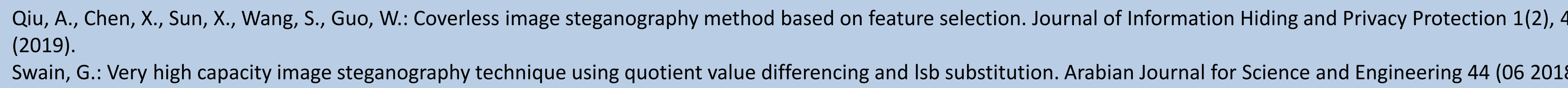

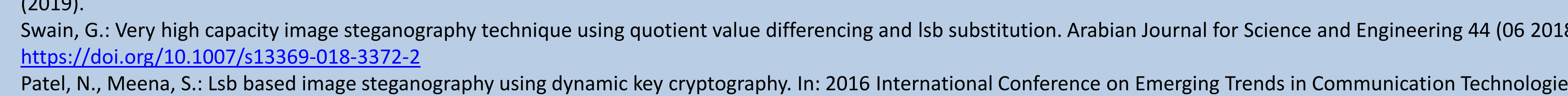

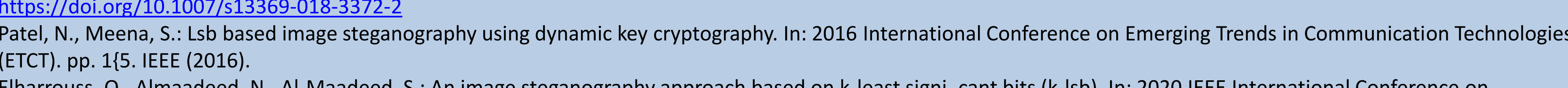
Elharrouss, O., Almaadeed, N., Al-Maadeed, S.: An image steganography approach based on k-least signi_cant bits (k-Is). In: 2020 IEEE International Conference on Xintao, D., Jia, K., Li, B., Guo, D., Zhang, E., Qin, C.: Reverrsible image steganography scheme based on a u-net structure. IEEE Access PP, 111 (01 2019). https://doio.org/10.1109/ACCESS.2019.2891247 